\title{
Dynamics of thin-walled element milling expressed by recurrence analysis
}

\author{
Rafal Rusinek • Kazimierz Zaleski
}

Received: 14 January 2015/ Accepted: 24 September 2015/Published online: 13 October 2015

(C) The Author(s) 2015. This article is published with open access at Springerlink.com

\begin{abstract}
This paper presents the results of experimental research on the stability of a milling process for producing a thin-walled part made of AL7075 aluminium alloy. The part was machined on a $\mathrm{CNC}$ milling machine with a decreasing wall thickness. The acceleration and cutting forces in the process were measured and analyzed to determine stability limit using classical stability diagrams as well as recurrence plots and recurrence quantification analysis.
\end{abstract}

Keywords Milling stability - Thin-walled element milling $\cdot$ Recurrence plot

\section{Introduction}

A relative motion between a cutting tool and a workpiece in cutting processes very often occurs as self-excited vibrations, also called chatter. Chatter is an undesired phenomenon because it deteriorates the finished surface and promotes the wear of tools and

R. Rusinek $(\bowtie)$

Department of Applied Mechanics, Lublin University of Technology, Nadbystrzycka 36, 20-618 Lublin, Poland e-mail: r.rusinek@pollub.pl

\section{K. Zaleski}

Department of Production Engineering, Lublin University of Technology, Nadbystrzycka 36, 20-618 Lublin, Poland e-mail: k.zaleski@pollub.pl components of cutting machines [19]. Moreover, chatter vibrations can cause the amplitude increase to a higher extent than in stable (vibration-free) cutting, which is particularly dangerous in practice [9]. The description of physical mechanisms that cause chatter phenomena can be found in [39]. Usually, the regenerative chatter (also called secondary chatter) is one of the most common causes of instability in the cutting processes. However, Grabec [14] and Wiercigroch et al. [40] showed that frictional effect is also important, because it leads to the so-called frictional or primary chatter. Generally, the problem of regenerative and frictional chatter is also discussed in [18, 31, 40]. From a practical point of view the choice of proper cutting conditions is very important because it can prevent chatter vibrations. In order to define a set of parameters which ensure stable cutting conditions the so called stability lobes diagrams (SLD) should be employed. SLDs help determine the value of acceptable cutting width (or depth) as a function of spindle speed. This is particularly important in high speed machining (HSM). HSM is often used in practice in order to increase process efficiency and reduce production costs. An in-depth overview of both selfexcited vibrations in cutting processes and methods for chatter prediction is given in [29].

Aluminium alloys are widely used in the aviation industry because of their low mass density. Although more and more thin-walled structural components are nowadays made of composite materials [35], some applications still require that these components be 
made of aluminum alloys which are typically used for relatively rigid thin-walled elements with complicated design. On the other hand, the machining of thinwalled components means removing even up to $90 \%$ of their volume. In order to do this effectively, high cutting depths and feed rates should be applied, which leads to an increase of cutting forces. If the forces are too high they can cause stability loss of a thin-walled workpiece. The literature provides a great deal of analytical, numerical and experimental methods for stability prediction. For instance, Altintas and Budak describe an analytical method for predicting milling stability lobes based on the mean of the Fourier series of the dynamic milling coefficients [2]. This method is fast but cannot predict additional stability regions and period doubling bifurcation for a low radial depth of cut [10]. To overcome this problem, a multi-frequency solution of chatter stability was developed by Budak and Altintas [6] and then extended by Merdol and Altintas [28].

One of the most popular numerical methods for chatter prediction is the Finite Element Method (FEM) [1, 13, 24, 37]. Bayly et al. [5] propose the use of a temporal finite element analysis for milling and interrupted turning [4]. Moreover, Voronov [37] and especially Adetoro et al. [1] propose an improved model of the classical milling process. The author of the work [1] takes into account the well-known stability model and supplements it with considerations about the nonlinearity of the cutting force coefficients and axial immersion angle as a function of the axial depth of cut. Besides, the authors indicate that thinwalled workpiece dynamics is nonlinear. The proposed model is validated and the theoretical findings show very good agreement with the experimental results [1]. Although, experimental results are very often compared with FEM results [3, 17], the problem of experimental signal analysis remains an open question. Different methods of signal analysis are applied in order to recognize chatter vibrations in cutting operations, including multifractal and wavelet approaches [38], multiscale entropy [23] HilbertHuang transform [20], recurrence analysis [22], flicker-noise spectroscopy [21] and audio signal analysis [32]. As it was mentioned before, the HSM method is also applied to thin-walled aluminium components for the aircraft industry to increase productivity [8]. Then, the flexibility of the tool and the workpiece, high spindle speeds and the inherent impact of nonlinearities in the milling process lead to complicated tool-workpiece interactions. In the paper [8] the method of delay space reconstruction is applied to show, that the workpiece motion is characterized by fractal geometry. The auto-bispectra suggests quadratic phase coupling among the spectral peaks associated with the cutter frequency. Finally, the authors propose a mechanics-based model with an impact so as to explain the obtained results. Their predictions agree well with the experimental observations. Arnaud et al. [3] presents the problem of modelling of machining vibrations of thin-walled aluminium workpieces at high productivity rate. These authors employ numerical simulations, FEM analysis and experimental tests in order to determine optimal cutting conditions. As a results, chatter frequencies are obtained. Moreover their study draws attention to the fact that the prediction of amplitudes in case of unstable milling is a very complex problem. Therefore, the simulations of thin-walled components machining require the applications of developed cutting force models including contact modelling and ploughing due to the variations in clearance angle during machining. In the machining process of thin-walled structure, it can be observed that the dynamic behaviour of the tool workpiece system depends on the tool position in the workpiece. This phenomenon and the problem of the influence of several modes on stability lobes are discussed in [36]. On the other hand, modal interaction can affect surface roughness in milling thin-walled structures [33].

The models of cutting processes are described by delay differential equations which may pose some problems in numerical calculations. To accelerate the integration procedure the semi or full discretization methods for prediction of milling stability are proposed in several papers [10]. The semi-discretization method, developed by Insperger and Stepan is an efficient numerical technique for stability analysis of linear delayed systems. However, Ding et al. [10] present a full-discretization method for prediction of milling stability which is even more effective. Insperger [16] proposes the so called act-and-wait concept for continuous-time control systems with feedback delay. The problem of chatter control is also discussed in [34] as well.

This paper focuses on the problem of milling stability of thin-walled element made of aluminium alloy 7075. A similar problem is discussed in [7], 
where the authors present a stability prediction method for the workpiece dynamics based on a structural modification technique. They consider a continuous change in the in-process workpiece structure during a machining cycle. With this method, the frequency response function of the workpiece is obtained by FEA only once, and it is continuously modified by depending on the workpiece volume removed during the cycle. The simulation results show good agreement with the experimental findings.

Here, in this paper an influence of wall thickness on process stability is valuated with an experiment. Next, the recurrence plot technique is applied to show various kinds of dynamical properties depending on wall thickness.

The primary objective of the paper is to determine milling stability expressed by means of the new index obtained from recurrence quantification analysis (RQA). Recurrence quantifiers are chosen for stability analysis because they enable finding a simple index for chatter recognition. Such index could be implemented later to a control system of chatter vibrations in any cutting processes.

\section{Experimental methodology}

The experimental investigations were conducted on the aluminium alloy Al7075 which was cut on the FV580A CNC milling machine equipped with the Fanuc control system. The experimental setup, shown schematically in Fig. 1 is consisted of two units: a modal analysis system and a dynamometer system. The former, comprised the PCB 086C03 modal hammer, the PCB 352B10 accelerometer and the data acquisition card NI9234, was used to measure the toolholder and the workpiece modal parameters. The latter was applied to measure three components of the total cutting force $\left(F_{x}, F_{y}, F_{z}\right)$ and acceleration in the $y$ direction. This part of the setup consisted of the Kistler 9257B piezoelectric dynamometer, the 5017B charge amplifier (Kistler) and the SCADAS Mobile LMS analyser was connected to a computer provided with the LMS TestXpress software. The cutting forces generated on the workpiece during the machining were measured by the dynamometer mounted on the milling machine underneath the workpiece. The corresponding force signals from the dynamometer were first transmitted to the charge amplifier next to

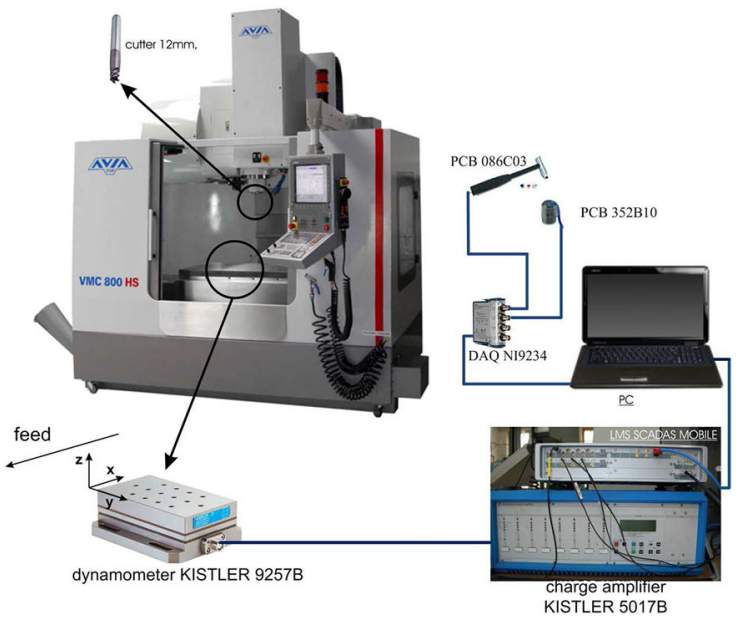

Fig. 1 Experimental setup

the LMS analyser and finally to the computer system. The sampling rate of data recorded during the test was set to $10 \mathrm{kHz}$. Although, the natural frequency of the tool is about $1.2 \mathrm{kHz}$, in some cases the workpiece frequency can even be $4 \mathrm{kHz}$. Therefore, the sampling rate was set to $10 \mathrm{kHz}$.

In order to measure vibrations during cutting, the PCB 352B10 accelerometer was mounted on the workpiece (Fig. 1). In the experiment, the solid carbide end mill FENES 6527 with a diameter of $12 \mathrm{~mm}$ and 2 cutting teeth was used to cut the workpiece made of aluminium alloy A17075. Other technological parameters included: the radial depth of cut $a_{e}=1 \mathrm{~mm}$, the axial depth of cut $a_{p}=22 \mathrm{~mm}$, the feed rate per tooth $f_{z}=0.1 \mathrm{~mm}$ and the spindle speed $n=20000 \mathrm{rpm}$. A scheme of the milling process is shown in Fig. 2. The experiment was

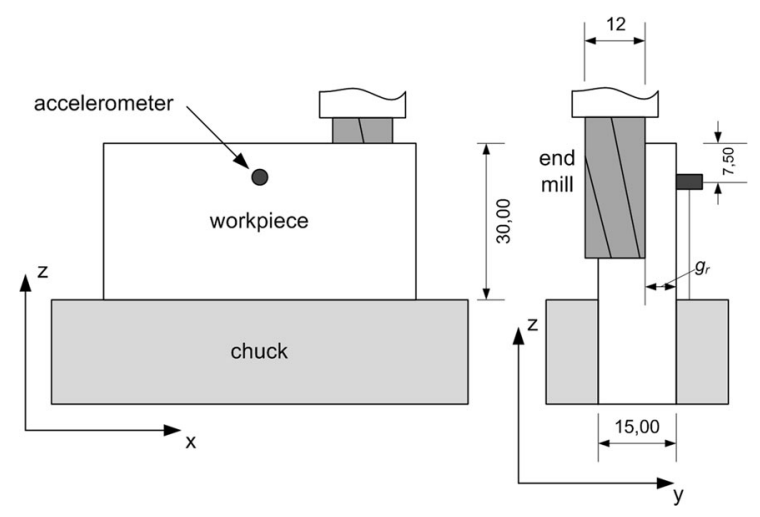

Fig. 2 Scheme of milling process 

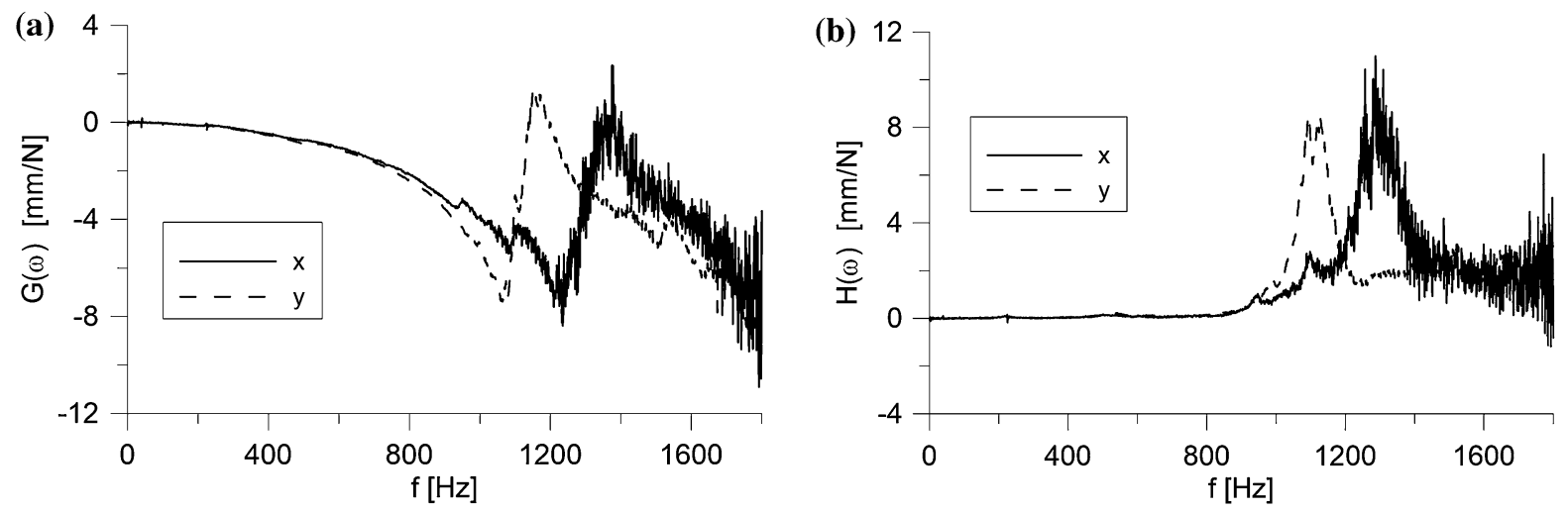

Fig. 3 FRF for spindle-end mill system, real part $\mathrm{G}(\omega)$ (a), imaginary part $\mathrm{H}(\omega)(\mathbf{b})$

Fig. 4 FRF for the thinwalled workpiece, real part $\mathrm{G}(\omega)(\mathbf{a})$, imaginary part $\mathrm{H}(\omega)(\mathbf{b})$
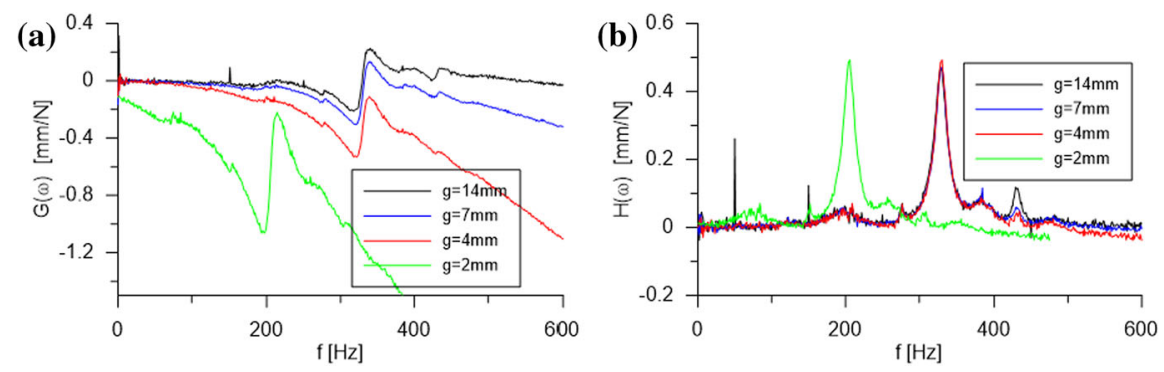

conducted with a decreasing wall thickness $(g)$ of the machined element, ranging from $g=14 \mathrm{~mm}$ to $g=2 \mathrm{~mm}$. In each tool pass the wall thickness was reduced by $1 \mathrm{~mm}$, which is the radial depth of cut $\left(a_{e}\right)$.

As it was mentioned above, the measurements were conducted in two steps. First, the impact test was performed to determine a stiffness coefficient, a natural frequency and a damping ratio of the spindletool system. The modal hammer (PCB 086C03) was used to excite the tool and then the resulting vibrations were measured by the low mass accelerometer (PCB 352B10) mounted at the tool's tip. Next, the same impact test was performed for the workpiece in order to measure its modal parameters. After each tool pass the impact test was repeated for the workpiece with a smaller wall thickness to obtain modal parameters as a function of wall thickness $(g)$. The modal parameters of the tool in the $x$ and $y$ directions and for the workpiece in the $y$ direction were exported in the form of a frequency response function (FRF, Figs. 3, 4). The function was then processed using the CutPro9 software to produce classical stability lobes diagrams for different wall thickness. The FRFs show a significant difference in the natural frequencies of the tool and the workpiece on decreasing the wall thickness. The modal parameters will be described in detail in the next section. In the experiment the three components of the cutting force and the acceleration in the $y$ direction were measured to analyse stability of the process by means of a new method based on a recurrence technique described in the next section.

\section{Classical stability analysis}

A classical stability analysis of the milling process for a thin-walled element made of Al7075 aluminium alloy was performed using the CutPro9 software, the results were then verified by investigating cutting forces and workpiece acceleration. Prior to the milling tests a stability lobes diagram (SLD) was generated with the CutPro9 using the FRF shown in Figs. 3 and 4 , in order to get the proper technological parameters and determine the critical depth of cut $\left(a_{p c}\right)$. The critical depth of cut is the highest value of cutting depth when the process remains stable regardless of the spindle speed. The modal parameters which were necessary to calculate the SLD were taken 
Fig. 5 Stability lobes diagram calculated for A17075 using CutPro ver. 9 software for

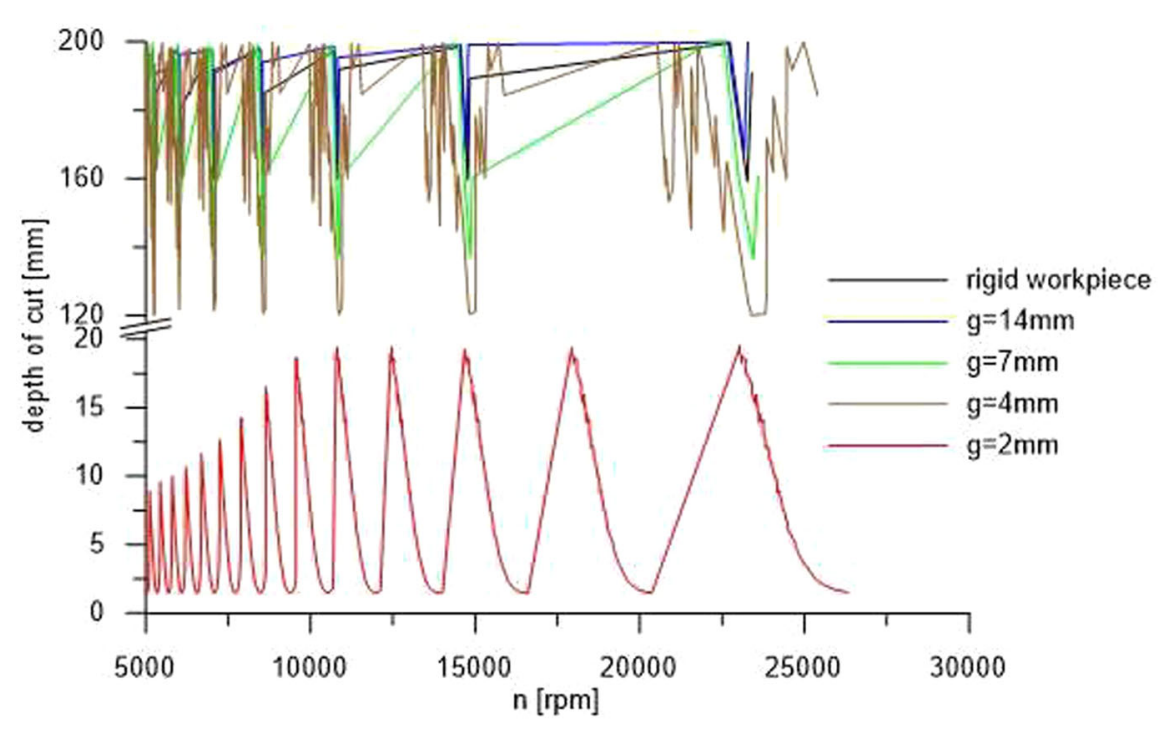

automatically from the $\mathrm{FRF}=\mathrm{G}(\omega)+j \mathrm{H}(\omega)$ (Figs. 3, 4). The real part $\mathrm{G}(\omega)$ contained information about the damping ratio, while the imaginary part $H(\omega)$ provided data about the natural frequency of the system. The resulting SLD is shown in Fig. 5. First, the workpiece was treated as a rigid body (the black curve), next as a flexible (thin-walled) element (the blue curve) with the initial wall thickness $g=14 \mathrm{~mm}$. The difference in the critical depth of cut $\left(a_{p c}\right)$ between the blue and black curves is negligible. In both cases $a_{p c}=160 \mathrm{~mm}$. This relatively high value of the axial critical depth of cut $\left(a_{p c}\right)$ resulted from a low radial depth of cut $\left(a_{e}\right)$, which was set to $1 \mathrm{~mm}$. Moreover, the tool was short and very stiff, therefore the stability limit $\left(a_{p c}\right)$ was high. After several tool passes (remembering that with each tool pass the wall thickness $g$ was decreased by $1 \mathrm{~mm}$ ) when the wall thickness $g=7 \mathrm{~mm}$, the critical depth of cut $a_{p c 7}$ in the SLD (Fig. 5) was $140 \mathrm{~mm}$, at $g=4 \mathrm{~mm}$ $a_{p c 4}=110 \mathrm{~mm}$, finally at $g=2 \mathrm{~mm} a_{p c 2}$ decreased to $1 \mathrm{~mm}$. In the milling experiment the three components of the total cutting force were measured together with the acceleration in the $y$ direction. This direction was chosen because of low workpiece stiffness. Examining the acceleration of vibrations measured on the workpiece during milling (Fig. 6a), it can be observed that the chatter vibrations significantly increase when the wall thickness $g<5 \mathrm{~mm}$, while the cutting forces (Fig. 6b-d) increase when $g<3 \mathrm{~mm}$. The differences between the wall thickness $(g)$ resulting from the analysis of the forces and the acceleration is caused by the fact that, thin-walled workpiece is so flexible that it escapes from the tool. Then, the forces decrease but the vibrations are higher. Given the above, it can be claimed that the acceleration signal is better than cutting forces for predicting instability. According to the SLD (Fig. 5), when the wall thickness $g=4 \mathrm{~mm}$ the critical depth of cut should be $a_{p c}=100 \mathrm{~mm}$, but the vibrations measured by the accelerometer at $a_{p}=22 \mathrm{~mm}$ are also high that the milling process cannot be treated as stable at all. Definitely, when $g<4 \mathrm{~mm}$ the process is unstable. Moreover, when $g=2 \mathrm{~mm}$ the measured acceleration is even $500 \mathrm{~g}$. Therefore, the correctness of the procedure for SLD prediction of the SLD implemented in CutPro9 poses serious doubts. The problem of SLD correctness is connected with a change in modal parameters of the workpiece which is particularly true with thin-walled elements. In the final test of the experiment, the workpiece mass decreased by $33 \%$, while the wall thickness decreased from 3 to $2 \mathrm{~mm}$. Therefore, the change in all modal parameters of the workpiece is a key aspect here. Figure 7 presents the changes in the natural frequency $\left(\omega_{n}\right)$, damping ratio $(\zeta)$, mass $\left(m_{w}\right)$ and stiffness $(k)$ as a function of wall thickness $(g)$. It is worth notifying that the natural frequency $\left(\omega_{n}\right)$ decreases when the wall thickness is small $(2 \mathrm{~mm})$. This is in accordance with the classical theory of beam and plates vibrations. At $g=2 \mathrm{~mm}$, the workpiece has lower natural frequency $\left(\omega_{n}\right.$, Fig. 7a). The stability limit (critical depth of cut) is very low because the damping ratio decreased 
Fig. 6 Acceleration (a) and cutting forces $F x(\mathbf{b}), F y(\mathbf{c})$, $\mathrm{Fz}$ (d) during the milling of 7075 alloy

Fig. 7 Change in modal parameters of the workpiece versus wall thickness $(g)$, natural frequency (a), damping ratio (b), mass (c), stiffness $(\mathbf{d})$
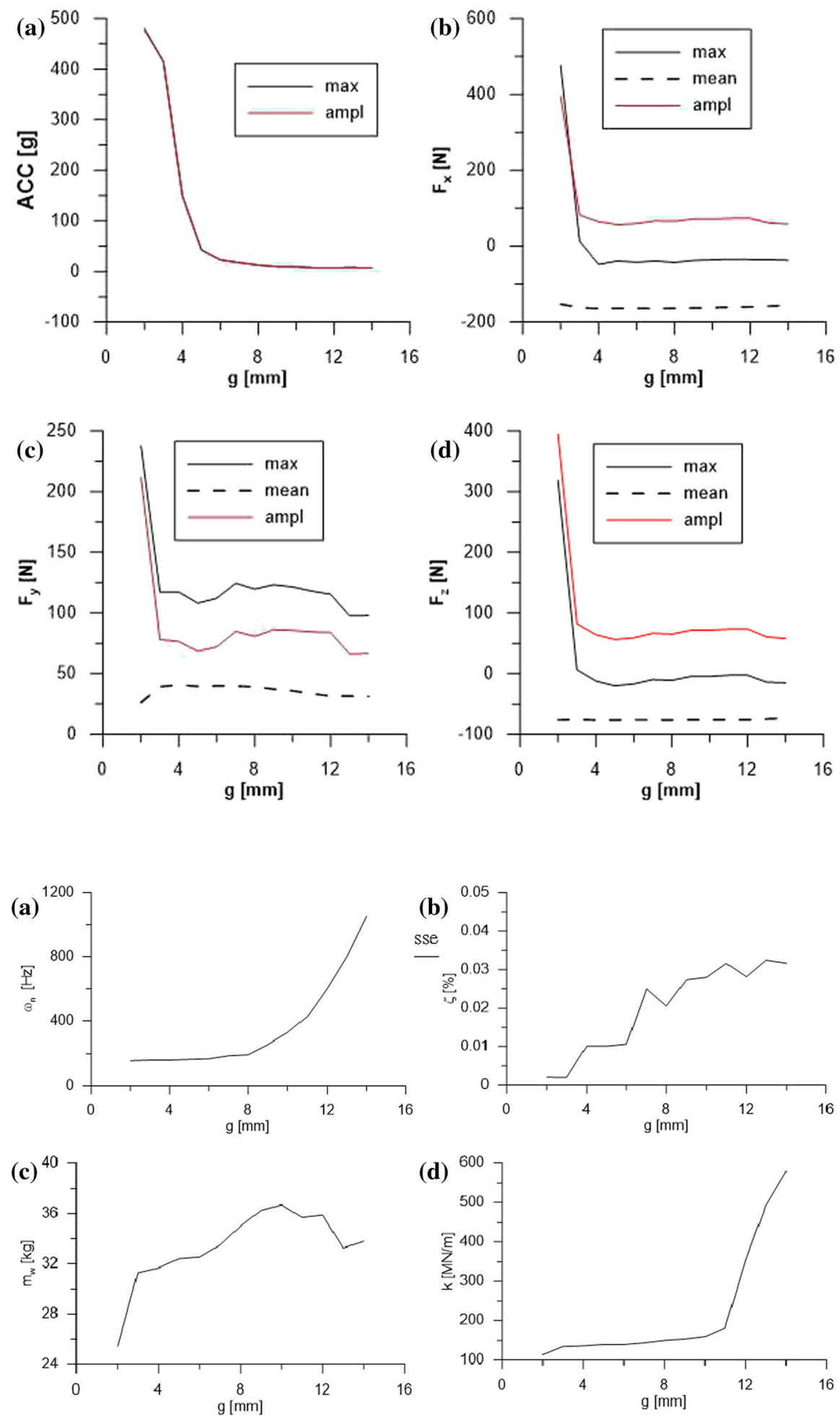
(Fig. 7b). Note, the damping ratio $(\zeta)$ is given in percentage as dimensionless coefficient, related to the natural frequency $\left(\omega_{n}\right)$. It means that the lower natural frequency, the lower damping in the system.

\section{Theory of recurrence analysis}

An extended analysis of stability and the experimental verification of process stability was performed using the recurrence plot (RP) technique. The RP approach provides a qualitative interpretation of hidden patterns of dynamical systems, based on phase space reconstruction. Phase space analysis methods are widely used for analysis of structural components applied in aircraft $[25,26]$. The recurrence plot technique was originally introduced by Eckmann [11]. The main idea of the phase space reconstruction assumes, that any time series $x_{i}$ can be presented as a delayed vector si in an $m$-dimensional space called 'the reconstructed phase space', as follows

$s_{i}=\left(x_{i}, x_{i}+d, x_{i+2 d}, \ldots, x_{i+(m-1) d}\right)$

here $m$ is the embedding dimension and $d$ means the time delay. Usually the embedding parameters, that is the time delay $(d)$ and embedding dimension $(m)$ can be estimated by means of the average mutual information function (AMI) and the false nearest neighbours method (FNN). The average mutual information (AMI) is plotted versus the time delay, and the adequate value of $d$ corresponds to the first local minimum. This procedure is an equivalent of an autocorrelation function for a linear case. If the delay is too long, then the coordinates are essentially independent and no information can be gained from the plot. If the delay is too short, then the reconstructed states differ not much and the points are scattered around the straight line. The false nearest neighbours function (FNN) is based on searching for an m-dimensional state space in which there are no false crossings of the trajectories. More information about the AMI and the FNN functions can be found in $[12,30]$. In the present work, the Tisean software [15] is used to obtain the embedding parameters. The recurrence plot technique is a graphical method designed for locating recurring patterns, a nonstationarity and structural changes. A recurrence plot is a graph which shows all the time instants at which the state of dynamical system recurs. The recurrence plot can be expressed as
$M_{i j}=\theta\left(\varepsilon-\left|s_{i}-s_{j}\right|\right)$

where $\theta$ is the Heaviside step function, $\varepsilon$ is a tolerance parameter (the threshold or neighbourhood size), $s_{i}$ and $s_{j}$ are delay vectors. If the trajectory in the reconstructed phase space returns at a time $i$ into the neighbourhood of $\varepsilon$, where it was before at the time $j$, then $M_{i j}=1$; otherwise $M_{i j}=0$. The results are plotted as black $\left(M_{i j}=1\right)$ and white $\left(M_{i j}=0\right)$ dots.

From a practical point of view, the RPs can be presented in a more useful and certainly more convenient form by the recurrence quantification analysis (RQA). The RQA is a method which quantifies the number and duration of recurrences of a dynamical system presented by its state space trajectory. The RQA measures were elaborated by Zbilut and Webber [41], then developed and introduced to the MATLAB software by Marwan [27]. The idea of RQA bases on the recurrence point density and the diagonal and vertical line structures of the recurrence plot. In this paper, the recurrence rate (RR), the determinism (DET), the laminaity (LAM), the laminarity to determinism ratio (LAM/DET), the divergence (DIV), the $\mathrm{L}$ and the $\mathrm{V}$ entropy $\left(L_{\text {ent }}, V_{\text {ent }}\right)$ are applied to investigate the milling stability. These measures are defined as follows:

- Recurrence rate (RR) is the density of recurrence points in a recurrence plot. In physical terms, RR denotes the probability that the system will recur

$$
\mathrm{RR}=\frac{1}{N^{2}} \sum_{i, j=1}^{N} M_{i, j}
$$

- Determinism (DET) is the fraction of recurrence points forming diagonal lines

$$
\mathrm{DET}=\frac{\sum_{l=l_{\min }}^{N} l P(l)}{\sum_{i, j=1}^{N} M_{i, j}},
$$

- Laminarity (LAM) is the fraction of recurrence points forming vertical lines. Vertical lines are typical for intermittency

$$
\mathrm{LAM}=\frac{\sum_{v=v_{\min }}^{N} v P(v)}{\sum_{v=1}^{N} v P(v)}
$$

- Laminarity to determinism ratio (LAM/DET)

$$
\mathrm{LAM} / \mathrm{DET}=\frac{\sum_{v=v_{\min }}^{N} v P(v)}{\sum_{v=1}^{N} v P(v)} / \frac{\sum_{l=l_{\min }}^{N} l P(l)}{\sum_{i, j=1}^{N} l P(l)}
$$


- Divergence (DIV) is an inverse of the longest diagonal length

$$
\mathrm{DIV}=\frac{1}{\max \left(\left\{l_{i} ; i=1 \ldots N_{l}\right\}\right)}
$$

- $L$-entropy $\left(L_{e n t}\right)$ is the Shannon's entropy of the diagonal line segment distribution

$$
L_{\text {ent }}=-\sum_{l=l \min }^{N} P(l) \ln P(l)
$$

- $L_{\max }$ is the length of the longest diagonal line

$$
L_{\max }=\max \left(\left\{l_{i} ; i=1 \ldots N_{l}\right\}\right)
$$

$\mathrm{P}(l)$ is the histogram of the lengths $l$ of the diagonal lines, $\mathrm{P}(v)$ is the histogram of the lengths $v$ of the vertical lines, $\mathrm{N}$ denotes the number of points on the phase space trajectory. The recurrence quantification analysis can provide useful information even for short and non-stationary data, where other methods fail. The RQA can be applied for various kinds of data to recognize dynamical behaviour [23]. Here the RQA is also applied to investigate milling process instability.

\section{Recurrence plots and recurrence quantification analysis}

The recurrence plots, presented in Figs. 8 and 9, show variations in the RPs patterns obtained in the stable $(g=12 \mathrm{~mm})$ and unstable $(g=2 \mathrm{~mm})$ milling. In Fig. 8 the RP is plotted from the cutting force $\left(F_{x}\right)$ while in Fig. 9 it is plotted from acceleration $(y)$. The embedding parameters $d$ and $m$ are calculated by the AMI and FNN functions, respectively. The proper value of the delay and the embedding dimension are given directly in Figs. 8 and 9. In stable milling the recurrence plots (Figs. 8a, 9a) show symptoms of regularity because the recurrence points extend the diagonal lines while in Figs. $8 \mathrm{~b}$ and $9 \mathrm{~b}$ no diagonal lines can be observed in the recurrence patterns. This is more visible in the case of accelerations (Fig. 9).
Fig. 8 Recurrence plots of $F x$ force when $g=14 \mathrm{~mm}$ (a), $g=2 \mathrm{~mm}$ (b)
Fig. 9 Recurrence plots of acceleration $(y)$ when $g=14 \mathrm{~mm}(\mathbf{a}), g=2 \mathrm{~mm}$ (b)
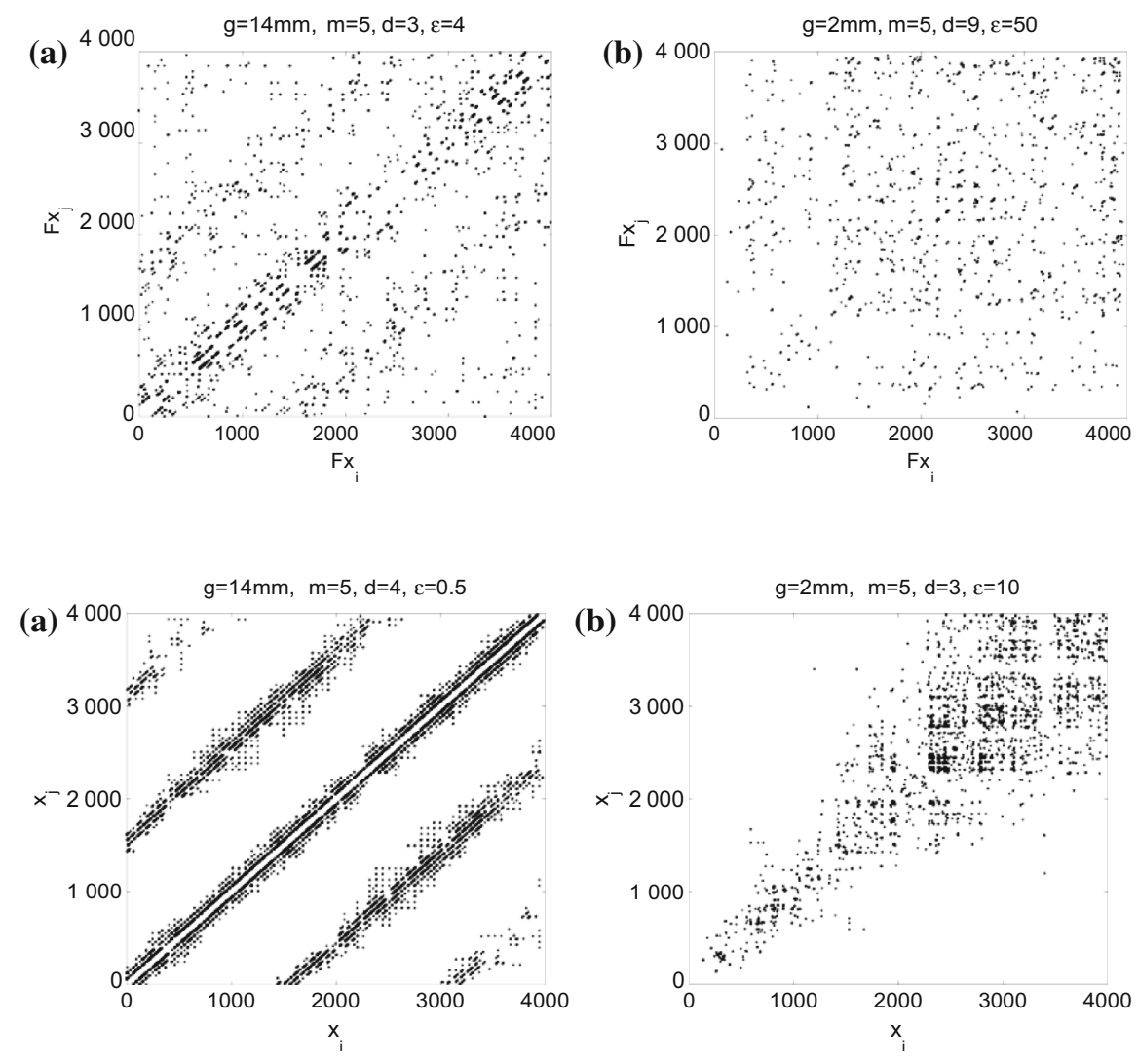
Fig. 10 Recurrence quantification analysis (RQA) of cutting force $(F x)$ : DET/RR (a), DET (b), LAM/DET (c), $L_{\text {ent }}(\mathbf{d})$, DIV (e), $L_{\max }(\mathbf{f})$
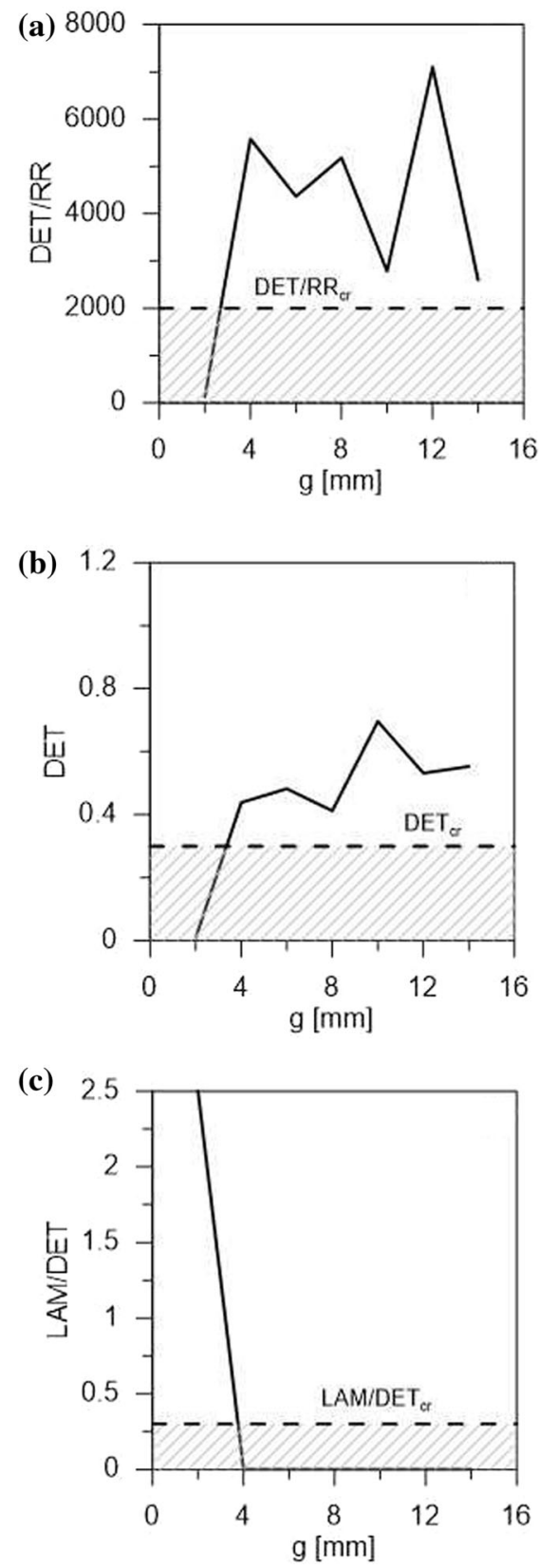
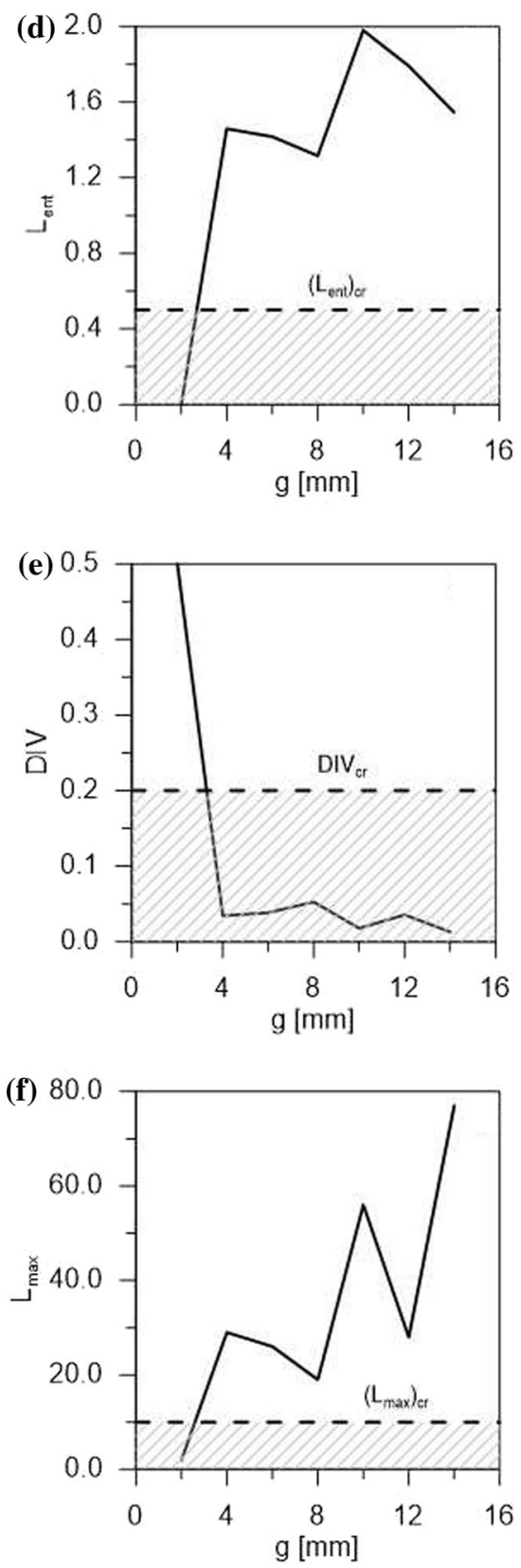

Thus, the analysis of the RPs can help to understand the process dynamics but it does enable finding the stability limit as this requires a great many of diagrams which can seem identical, especially near the stability limit. For this reason, practical implementation of RP is limited. Therefore, the RQA is much better to this end because it can give a direct and simple index of stability. Here, in our research DET/RR, DET, LAM/ DET, $L_{\text {ent }}$, DIV and $L_{\max }$ were analysed (Fig. 10). All the presented indexes, with the exception of LAM/ DET and DIV, exhibit a decrease when $g<4 \mathrm{~mm}$, while LAM/DET and DIV increases when $g<4 \mathrm{~mm}$. Thus, the RQA indexes can be used as stability indexes of milling process, provided that the proper values of critical indexes are found.

The critical indexes of RQA should be estimated in an experiment, for every cutting operation individually. In the experiment reported in this paper, the critical 
value of indexes were: $\mathrm{DET} / \mathrm{RR}_{c r}=2000, \mathrm{DET}_{c r}=$ $0.3, \mathrm{LAM}_{\mathrm{DET}} \mathrm{D}_{r}=0.3,\left(L_{e n t}\right)_{c r}=0.5, \mathrm{DIV}_{c r}=0.2$, $\left(L_{\max }\right)_{c r}=10$. If LAM/DET or DIV is smaller than the critical ones the milling process is stable. As for the other indexes, they should be set higher than critical ones to ensure that the process is stable.

\section{Discussion and conclusions}

This study investigated the problem of stability of the milling process for producing a thin-walled components. The stability limit estimated with the commercial software CutPro ver. 9 is not accurate either for the workpiece treated as a rigid body or even for the flexible workpiece described by the modal parameters taken from the impact test. The results of the experiment performed on the milling machine reveal that stability loss occurs much earlier than suggested by the CutPro9 module, despite the fact that the modal parameters of both the tool and the workpiece were measured after every tool pass and implemented in the programme for calculating stability diagrams. This means that the applied software is not suitable for investigating milling process for low mass elements (like thin-walled components) where the mass reduction is substantially high compared to the initial mass of the workpiece. However, undoubtedly, the main advantage of the CutPro software is the fact that it enables producing the SLD prior to running a cutting process (test). Stability loss is more visible in the cutting force signals and especially in the acceleration of vibrations. However, the acceleration signal indicates that a loss of stability is earlier (i.e. for a lower value of wall thickness) than the force signals suggest. This observation is in accordance with the experiment. The tests performed with the modal hammer let us determine the modal parameters of the workpiece which are changeable during the experiment. The decrease of the critical depth of cut with wall thickness is directly connected with a decrease in the damping ratio.

The recurrence diagrams and the method of phase space reconstruction method can determine stability loss of the milling process and provide a deeper insight into the system dynamics. From a practical point of view, however, these methods are not a convenient way of estimating stability limits. The use of recurrence quantification analysis is much better in this regard. When the process approaches instability, the stability index indicates that some technological parameters of the cutting process should be modified to prevent chatter. Therefore, we need the simplest numerical indexes. The reconstructed phase space analysis gives a deeper insight into process dynamics but the application of this analysis for the purpose of this paper is difficult. That is why, it was necessary to find a proper index which could be implemented later for chatter control system. The production of thinwalled parts, that are widely used in aircraft industry, is only one example of RQA applications. The selection of such RQA indexes as DET/RR, DET, LAM/DET, $L_{\text {ent }}$, DIV and $L_{\max }$ was dedicated by the fact that they exhibit the exact moment when a thin walled element losses stability in milling. In the future the RQA indexes could be applied to control the stability of cutting processes online. RQA is an alternative method of finding stability limit. Sometimes, it is simpler to calculate statistical parameters of force or acceleration signal but, RP and RQA give deeper insight in system dynamics.

Summing up, several conclusions can be dawn:

- the variation of modal parameters in the cutting process for low mass (thin walled) elements is crucial for stability diagram prediction. Modal mass, stiffness and damping ratio are essentially important, too;

- the proposed stability indexes based on recurrence quantification analysis can be used for determination of the stability limit if the critical (threshold) value is properly defined,

- the critical value of stability index should be estimated individually for every cutting operations.

Acknowledgments Financial support of Structural Funds in the Operational Programme-Innovative Economy (IE OP) financed from the European Regional Development FundProject "Modern material technologies in aerospace industry", POIG.01.01.02-00-015/08-00 is gratefully acknowledged.

Open Access This article is distributed under the terms of the Creative Commons Attribution 4.0 International License (http:// creativecommons.org/licenses/by/4.0/), which permits unrestricted use, distribution, and reproduction in any medium, provided you give appropriate credit to the original author(s) and the source, provide a link to the Creative Commons license, and indicate if changes were made. 


\section{References}

1. Adetoro O, Sim W, Wen P (2010) An improved prediction of stability lobes using nonlinear thin wall dynamics. J Mater Process Technol 210(6-7):969-979

2. Altintas Y, Budak E (1995) Analytical prediction of stability lobes in milling. CIRP Ann Manuf Technol 44(1):357-362. doi:10.1016/S0007-8506(07)62342-7

3. Arnaud L, Gonzalo O, Seguy S, Jauregi H, Peigne G (2011) Simulation of low rigidity part machining applied to thinwalled structures. Int J Adv Manuf Technol 54(5-8):479-488

4. Bayly PV, Halley JE, Mann BP, Davies MA (2003) Stability of interrupted cutting by temporal finite element analysis. J Manuf Sci Eng 125(2):220-225. doi:10.1115/1.1556860

5. Bayly PV, Mann BP, Schmitz TL, Peters DA, Stepan G, Insperger T (2002) Effects of radial immersion and cutting direction on chatter instability in end-milling. In: ASME 2002 international mechanical engineering congress and exposition, pp 351-363. doi:10.1115/IMECE2002-39116

6. Budak E, Altintaş Y (1998) Analytical prediction of chatter stability in milling — part I: general formulation. J Dyn Syst Meas Contrl 120(1):22. doi:10.1115/1.2801317

7. Budak E, Tunc LT, Alan S, Ozguven HN (2012) Prediction of workpiece dynamics and its effects on chatter stability in milling. CIRP Ann Manuf Technol 61(1):339-342

8. Davies MA, Balachandran B (2000) Impact dynamics in milling of thin-walled structures. Nonlinear Dyn 22(4):375-392

9. Davies MA, Duttrer B, Pratt JR, Schaut AJ, Bryan JB (1998) On the dynamic of high-speed milling with long, Slender Endmills. Ann CIRP 47(1):55-61

10. Ding Y, Zhu L, Zhang X, Ding H (2010) A full-discretization method for prediction of milling stability. Int $\mathbf{J}$ Mach Tools Manuf 50(5):502-509. doi:10.1016/j. ijmachtools.2010.01.003

11. Eckmann JP, Kamphorst SO, Ruelle D (1987) Recurrence plots of dynamical systems. Europhys Lett (EPL) 4(9):973-977. doi:10.1209/0295-5075/4/9/004

12. Fraser AM (1989) Reconstructing attractors from scalar time series: a comparison of singular system and redundancy criteria. Phys D 34(3):391-404

13. Gang L (2009) Study on deformation of titanium thin-walled part in milling process. J Mater Process Technol 209(6):2788-2793

14. Grabec I (1986) Chaos generated by the cutting process. Phys Lett A A117:384-386

15. Hegger R, Kantz H, Schreiber T (1999) Practical implementation of nonlinear time series methods: the TISEAN package. Chaos 9(2):413-435

16. Insperger $\mathrm{T}$ (2006) Act-and-wait concept for continuoustime control systems with feedback delay. IEEE Trans Control Syst Technol 14(5):974-977. doi:10.1109/TCST. 2006.876938

17. Izamshah R, Mo J, Ding S (2012) Hybrid deflection prediction on machining thin-wall monolithic aerospace components. Proc Inst Mech Eng Part B J Eng Manuf 226(B4):592-605

18. Kecik K, Rusinek R, Warminski J (2013) Modeling of highspeed milling process with frictional effect. Proc Inst Mech Eng Part K J Multi-body Dyn 227(1):3-11. doi:10.1177/ 1464419312458636
19. Lipski J, Litak G, Rusinek R, Szabelski K, Teter A, Warminski J, Zaleski K (2002) Surface quality of a work material's influence on the vibrations of the cutting process. J Sound Vib 252(4):729-737

20. Litak G, Kecik K, Rusinek R (2013) Cutting force response in milling of Inconel: analy- sis by wavelet and HilbertHuang transforms. Latin Am J Solids Struct 10(1):133-140. doi:10.1590/S1679-78252013000100013

21. Litak G, Polyakov YS, Timashev SF, Rusinek R (2013) Dynamics of stainless steel turning: analysis by flickernoise spectroscopy. Phys A 392(23):6052-6063. doi:10. 1016/j.physa.2013.07.079

22. Litak G, Rusinek R (2012) Dynamics of a stainless steel turning process by statistical and recurrence analyses. Meccanica 47(6):1517-1526. doi:10.1007/s11012-0119534-x

23. Litak G, Syta A, Rusinek R (2011) Dynamical changes during composite milling: recurrence and multiscale entropy analysis. Int J Adv Manuf Technol 56(5-8):445-453

24. Mane I, Gagnol V, Bouzgarrou B, Ray P (2008) Stabilitybased spindle speed control during flexible workpiece highspeed milling. Int J Mach Tools Manuf 48(2):184-194

25. Manoach E, Samborski S, Mitura A, Warminski J (2012) Vibration based damage detection in composite beams under temperature variations using Poincaré maps. Int J Mech Sci 62(1):120-132. doi:10.1016/j.ijmecsci.2012.06.006

26. Manoach E, Warminski J, Mitura A, Samborski S (2012) Dynamics of a composite Timoshenko beam with delamination. Mech Res Commun 46:47-53. doi:10.1016/j. mechrescom.2012.08.008

27. Marwan N, Kurths J (2002) Nonlinear analysis of bivariate data with cross recurrence plots. Phys Lett A 302:299-307

28. Merdol SD, Altintas Y (2004) Multi frequency solution of chatter stability for low immersion milling. J Manuf Sci Eng Trans Asme 126(3):459-466

29. Quintana G, Ciurana J (2011) Chatter in machining processes: a review. Int J Mach Tools Manuf 51(5):363-376. doi:10.1016/j.ijmachtools.2011.01.001

30. Rusinek R (2010) Cutting process of composite materials: an experimental study. Int J Non-Linear Mech 45:458-462

31. Rusinek R, Szabelski K, Warminski J (2004) Influence of the workpiece profile on the self-excited vibrations in a metal turning process. In: Radons G, Neugebauer R (eds) Nonlinear dynamics of production systems. Wiley-VCH, Weinheim, pp 153-167

32. Schmitz TL, Medicus K, Dutterer B (2002) Exploring onceper-revolution audio signal variance as a chatter indicator. Mach Sci Technol 6(2):215-233. doi:10.1081/MST120005957

33. Seguy S, Dessein G, Arnaud L (2008) Surface roughness variation of thin wall milling, related to modal interactions. Int J Mach Tools Manuf 48(3-4):261-274. doi:10.1016/j. ijmachtools.2007.09.005

34. Shi H, Hu H (2007) Novel on-line monitoring method of cutting chatter based on neural network pattern recognition technique. In: Second international conference on innovative computing, information and control (ICICIC 2007), p. 565. doi:10.1109/ICICIC.2007.411

35. Teter A, Debski H, Samborski S (2014) On buckling collapse and failure analysis of thin-walled composite lipped- 
channel columns subjected to uniaxial compression. Thin Walled Struct 85:324-331

36. Thevenot V, Arnaud L, Dessein G, Cazenave-Larroche G (2006) Integration of dynamic behaviour variations in the stability lobes method: 3D lobes construction and application to thin-walled structure milling. Int J Adv Manuf Technol 27(7-8):638-644. doi:10.1007/s00170-004-2241-1

37. Voronov S, Kiselev I (2011) Dynamics of flexible detail milling. Proc Inst Mech Eng Part K J Multi-body Dyn 225(K4):299-309

38. Wang L, Liang M (2009) Chatter detection based on probability distribution of wavelet modulus maxima. Robot
Comput Integr Manuf 25(6):989-998. doi:10.1016/j.rcim. 2009.04.011

39. Wiercigroch M, Budak E (2001) Sources of nonlinearities, chatter generation and suppression in metal cutting. Philos Trans R Soc A Math Phys Eng Sci 359(1781):663-693

40. Wiercigroch M, Krivtsov AM (2001) Frictional chatter in orthogonal metal cutting. Philos Trans R Soc Soc Lond A Math Phys Eng Sci 359:713-738

41. Zbilut JP, Webber CLJ (1992) Embeddings and delays as derived from quantification of recurrence plots. Phys Lett A 171:199-203 